\title{
Parental perceptions and childhood overweight/obesity: A population-based study among school children in Japan
}

\author{
Takako Shirasawa $^{1^{*}}$, Hirotaka Ochiai ${ }^{1}$, Tadahiro Ohtsu ${ }^{1}$, Rimei Nishimura $^{2}$, Takaya Shimizu $^{1}$, \\ Hiromi Hoshino ${ }^{1}$, Naoko Tajima ${ }^{3}$, Akatsuki Kokaze ${ }^{1}$ \\ ${ }^{1}$ Department of Public Health, Showa University School of Medicine, Tokyo, Japan; \\ ${ }^{*}$ Corresponding Author: shirasawa@med.showa-u.ac.jp \\ ${ }^{2}$ Division of Diabetes, Metabolism and Endocrinology, Department of Internal Medicine, Jikei University School of Medicine, To- \\ kyo, Japan \\ ${ }^{3}$ Jikei University School of Medicine, Tokyo, Japan
}

Received 12 June 2012; revised 6 July 2012; accepted 13 July 2012

\section{ABSTRACT}

The lifestyle of children is primarily controlled by their parents, and that childhood obesity is related to family variables. The aim of this study was to investigate the relationship between parental perceptions on childhood obesity and their child's overweight/obesity in Japan. The study subjects were 3168 school children (aged 9 or 10 years) and their parents in the town of Ina, Saitama Prefecture, Japan, between 2002 and 2009. Information regarding parental perceptions about childhood obesity and the lifestyle factors of their children was collected using selfadministered questionnaires completed by the participants and their parents. Childhood overweight was defined according to the International Obesity Task Force cutoffs. Parental perceptions on childhood obesity were significantly associated with their child's overweight status. Specifically, low perceptions regarding childhood obesity significantly increased the odds ratio (OR) for the child's overweight status (OR: 1.86, 95\% Cl: 1.32 - 2.62). However, when the analysis was limited to children with both parents being overweight, there was no statistically significant difference between levels of parental perceptions and the overweight status of their children. Accordingly, the present study suggests that, when both parents are obese, not only is raising parental awareness important, but also encouraging parents to prevent themselves from becoming overweight, leads to improvements in the lifestyle habits of children, which may contribute to the prevention of childhood overweight.
Keywords: Children; Childhood Obesity; Parental Perceptions; Parental Obesity; Japan

\section{INTRODUCTION}

The prevalence of childhood obesity and overweight has been increasing worldwide [1]. In Japan, the prevalence of obese boys and girls increased from $6.1 \%$ and $7.1 \%$, respectively, between 1976 and 1980 to $11.1 \%$ and $10.2 \%$, respectively, between 1996 and 2000 [2]. Obesity is associated with the risk factors and indicators of cardiovascular disease and metabolic syndrome [3]. Moreover, some studies have shown that these indicators may be persistent from childhood and adolescence to young adulthood [4,5]. Therefore, it is very important to prevent childhood obesity and overweight.

The causes of overweight and obesity among children can be divided into genetic [6] and lifestyle factors, which include physical activity and eating habits $[7,8]$. Several studies have shown that the lifestyle of children is primarily controlled by their parents, and that childhood obesity is related to family variables [9-17].

Among the family variables, parental perceptions and attitudes regarding childhood obesity have been reported to be associated with their child's overweight or obesity [12,18-20]. A previous study reported that parents of overweight children do not recognize their child as overweight and are not aware of their overweight status [18]. Thus, low parental perceptions of their child's overweight or obesity are unlikely to provide a healthy lifestyle or environment for their children [12]. Although some studies have been conducted on parental perceptions of their child's body types or weight $[19,20]$, little is known about the relationship between parental perceptions on childhood obesity and their child's overweight or obese status. If associations between parental percep- 
tions on childhood obesity and the child's overweight/ obesity status are revealed, this evidence may help in the prevention of childhood overweight/obesity. Therefore, it is important to assess the levels of parental perceptions on childhood obesity and evaluate the association between parental perceptions and their child's overweight status.

Accordingly, the aim of the present study was to investigate the relationship between parental perceptions on childhood obesity and childhood overweight in a population-based study among Japanese school children.

\section{METHODS}

In addition to the annual national health check-ups performed in accordance with the School Health Law of Japan, the town of Ina in the Saitama Prefecture, Japan, has been conducted a unique health promotion program for the prevention of childhood lifestyle-related diseases since 1994. The program consists of a questionnaire survey, as well as blood and physical examinations, of fourth and seventh graders. Several studies describing this program have been reported [21-24], and the present study was also conducted as part of the program.

\subsection{Subjects}

The study subjects were all fourth graders (aged 9 - 10 years) between 2002 and 2009 in the town of Ina. Written, informed consent was obtained from the parent or guardian of each subject. The study protocol was approved by the Medical Ethics Committee of Showa University School of Medicine.

\subsection{Anthropometric Measurements}

During the annual school health examination, all participants were asked to remove their shoes and socks, after which their height and body weight were measured in units of $0.1 \mathrm{~cm}$ and $0.1 \mathrm{~kg}$, respectively, while they were wearing light clothing. Body mass index (BMI) was calculated as body weight $(\mathrm{kg})$ divided by the square of the height $\left(\mathrm{m}^{2}\right)$.

\subsection{Questionnaire Survey}

A self-administered questionnaire was distributed to each study participant and the parent (father or mother) or guardian by the class teachers one week before the annual school health examination. It was completed by the child and the parent or guardian at home, and was then collected on the day of the health examination.

The following information was collected from each participant: sex, age, exercise aside from physical education class (daily, sometimes, or none), eating of snacks after dinner (always, often, seldom, or none), eating speed (fast, medium, slow), eating while doing something else (Yes or No), and chewing thoroughly (Yes or No).

The parent or guardian of each participant was asked to answer the following questions regarding the participant: birth weight, frequency of eating breakfast (daily, sometimes, or none), dinnertime (regular or irregular), bedtime (regular or irregular), and wake-up time (regular or irregular). He or she was also asked to provide the following information regarding the family: "whether the child was the only child", "the age, height, weight, and employment of the parents", and "parental perceptions on childhood obesity". The birth weights of the participants were categorized into five groups: $<2000 \mathrm{~g}, 2000$ 2499 g, $2500-2999$ g, $3000-3499$ g, and $\geq 3500$ g. The frequency of eating snacks after dinner was categorized into two groups: Yes (always or often) and No (seldom or none). The frequency of eating breakfast was categorized into two groups: skipping breakfast (sometimes or none) and not skipping breakfast (daily). Information regarding parental perceptions on childhood obesity was obtained from five answer options: "I think of childhood obesity as a serious problem", "I think of it as a problem", "I cannot say whether childhood obesity is a problem or not", "I don't think of it as a serious problem", and "I don't think of it as a problem". Those who thought that childhood obesity was a serious problem or a problem were regarded as those whose perceptions were high or moderate, respectively. Respondents that chose "I cannot say whether childhood obesity is a problem or not", "I don't think of it as a serious problem", or "I don't think of it as a problem" were grouped as those whose perceptions were low, as the numbers in these three categories were small.

\subsection{Definition of Overweight}

Childhood overweight (including obesity) was determined according to the age- and sex-specific cutoff points proposed by the International Obesity Task Force [25]. Obesity in parents was regarded as a BMI $\geq 25$ $\mathrm{kg} / \mathrm{m}^{2}$, according to the World Health Organization criteria [26].

\subsection{Statistics}

The chi-square test or unpaired t-test was used to compare various characteristics between the overweight and non-overweight groups. The relationship between parental perceptions on childhood obesity and the child's overweight status was investigated using a logistic regression model. Crude odds ratios (ORs) for overweight and the $95 \%$ confidence interval $(95 \% \mathrm{CI})$ were initially calculated, and then adjusted for potential confounders. Variables that were different between the overweight and non-overweight groups with a $P$ value $<0.05$ were con- 
sidered potential confounders. A $P$ value $<0.05$ was considered as statistically significant. Data analyses were performed using the SPSS 16.0J (IBM, Chicago, IL, USA).

\section{RESULTS}

Of the 3179 subjects, 11 were excluded from the analysis due to refusal to take part in the study or school absence. Thus, data from a total of 3168 subjects (1645 boys and 1523 girls) were analyzed (participation rate: 99.7\%). The characteristics of parents (fathers and mothers) by the overweight status of their children are shown in Table 1. Parents with overweight children had significantly higher BMI than those with non-overweight children $(P<0.001)$. Children with one or both parents being overweight were more likely to be in the overweight group $(P<0.001)$. The employment status of the parents (fathers and mothers) was not related to the child's overweight. There was a statistically significant difference between parental perceptions on childhood obesity and the child's overweight status $(P<0.001)$.

Table 2 shows the characteristics of overweight and non-overweight children. The proportion of boys was significantly higher in the overweight group than in the non-overweight group. There was a statistically significant difference between the overweight and non-overweight children groups with respect to birth weight. Only children were more likely to be in the overweight group. Children who did not exercise, ate quickly, or did not chew thoroughly were more likely in the overweight group. The crude and adjusted ORs of parental obesity or perceptions on childhood obesity for their child's overweight status were calculated (Table 3).

The results showed that when one (OR: 1.83 , 95\% CI: $1.43-2.34)$ or both $(5.54,3.51-8.73)$ parents are obese, the ORs for childhood overweight significantly increased compared to when both parents were non-obese. Moreover, moderate $(1.52,1.21$ - 1.90) and low (1.86, 1.32 2.62) perceptions regarding childhood obesity resulted in significantly increased ORs for childhood overweight, compared to high perceptions.

Additionally, adjusted ORs of parental perceptions on childhood obesity for their child's overweight status were calculated according to the obesity status of the parents (Table 4). When one or both parents were non-obese, moderate or low perceptions regarding childhood obesity significantly increased the ORs for childhood overweight, when compared to high perceptions regarding childhood obesity. However, there was no statistically significant difference between parental perceptions on childhood obesity and their child's overweight when both parents were obese.

\section{DISCUSSIONS}

In the present study, the association between parental perceptions on childhood obesity and the overweight status of their children was examined. It was found that childhood overweight status was significantly associated with parental perceptions on childhood obesity. In particular, low or moderate perceptions regarding childhood obesity significantly increased the OR for their child's overweight status.

Our results indicated that there was a statistically significant difference in some baseline characteristics, such as birth weight and the number of siblings, between the overweight and non-overweight children. For example, children without siblings were more likely to be overweight. These observations were consistent with the results of previous studies [27,28]. Moreover, many studies have reported that lifestyle factors, such as physical inactivity and eating habits, are associated with childhood and adolescent obesity $[8,12,17,29]$. Therefore, we adjusted for these factors, which were significantly associated with children's overweight status in the analysis, to evaluate the relationship between parental perceptions on childhood obesity and their child's overweight status.

In the present study, parental obesity significantly increased the ORs for overweight in their children, which was consistent with previous studies [30-32]. Furthermore, in our study, low perceptions regarding childhood obesity significantly increased the OR for the child's overweight status. Low parental perceptions regarding childhood obesity may be due to a lower awareness of what constitutes a healthy lifestyle [33], which in turn, may be a risk factor for childhood overweight. Thus, an effective measure against childhood overweight may be an implementation of health education programs that aim to increase the knowledge of parents with low perceptions regarding childhood obesity.

Additionally, low parental perceptions regarding childhood obesity significantly increased the ORs for their child's overweight status, particularly when one or both parents are non-obese. These results suggest that parental perceptions regarding childhood obesity affect the overweight status of their children when neither or one parent was obese. Conversely, there were no significant increases in OR found when both parents were obese, suggesting that the levels of parental perceptions may not have a substantial effect on the overweight status of their children when both parents are obese. Both BMI and the health behaviors of children were reported to be associated with family variables, such as parental overweight or obesity, which also indicated a greater risk of obesity in offspring and should be recognized as a marker of families that are at risk [31]. A previous study showed that, when both parents were obese, parental attempts to improve their own lifestyle habits affected the living environment of their children, and resulted in an improvement in their child's overweight status [21]. Accordingly, 
Table 1. Parental characteristics according to the overweight status of study participants.

\begin{tabular}{|c|c|c|c|}
\hline & Non-overweight $(n=2683)$ & Overweight $(n=485)$ & $P^{\mathbf{b}}$ \\
\hline \multicolumn{4}{|l|}{ Father } \\
\hline Age (years) & $40.6 \pm 5.2$ & $40.9 \pm 5.4$ & 0.217 \\
\hline Height (cm) & $171.3 \pm 5.4$ & $170.9 \pm 6.2$ & 0.201 \\
\hline Weight (kg) & $68.8 \pm 9.7$ & $72.4 \pm 11.5$ & $<0.001$ \\
\hline BMI $\left(\mathrm{kg} / \mathrm{m}^{2}\right)$ & $23.4 \pm 2.9$ & $24.8 \pm 3.4$ & $<0.001$ \\
\hline \multicolumn{4}{|l|}{ BMI percentile } \\
\hline$<50$ th & 52.4 & 38.0 & $<0.001$ \\
\hline $50-74$ th & 24.9 & 24.3 & \\
\hline $75-84$ th & 9.5 & 13.0 & \\
\hline $85-94$ th & 9.2 & 13.9 & \\
\hline$\geq 95$ th & 4.0 & 10.8 & \\
\hline \multicolumn{4}{|l|}{ Mother } \\
\hline Age (years) & $38.2 \pm 4.2$ & $38.3 \pm 4.6$ & 0.739 \\
\hline Height (cm) & $158.0 \pm 5.2$ & $158.2 \pm 5.3$ & 0.357 \\
\hline Weight (kg) & $52.4 \pm 7.3$ & $57.1 \pm 9.8$ & $<0.001$ \\
\hline BMI $\left(\mathrm{kg} / \mathrm{m}^{2}\right)$ & $21.0 \pm 2.7$ & $22.8 \pm 3.5$ & $<0.001$ \\
\hline \multicolumn{4}{|l|}{ BMI percentile } \\
\hline$<50$ th & 53.3 & 31.5 & $<0.001$ \\
\hline $50-74$ th & 24.9 & 25.3 & \\
\hline $75-84$ th & 9.5 & 14.4 & \\
\hline $85-94$ th & 8.7 & 16.2 & \\
\hline$\geq 95$ th & 3.6 & 12.6 & \\
\hline \multicolumn{4}{|l|}{ Parental obesity $(\%)^{\mathrm{a}}$} \\
\hline Non-obese & 67.7 & 47.1 & $<0.001$ \\
\hline Either parent obese & 29.5 & 41.5 & \\
\hline Both parents obese & 2.8 & 11.5 & \\
\hline \multicolumn{4}{|c|}{ Parental employment (\%) } \\
\hline Father & 99.4 & 98.8 & 0.198 \\
\hline Mother & 54.3 & 58.7 & 0.100 \\
\hline \multicolumn{4}{|c|}{ Parental perceptions on childhood obesity (\%) } \\
\hline High & 45.4 & 33.4 & $<0.001$ \\
\hline Moderate & 44.9 & 52.4 & \\
\hline Low & 9.6 & 14.2 & \\
\hline
\end{tabular}

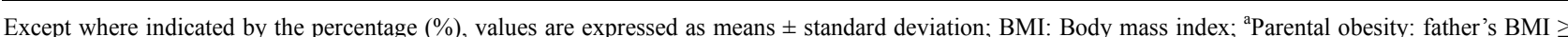
$25 \mathrm{~kg} / \mathrm{m}^{2}$ and/or mother's BMI $\geq 25 \mathrm{~kg} / \mathrm{m}^{2}$; ${ }^{b}$ Unpaired t-test or chi-squared test. 
Table 2. Characteristics of non-overweight and overweight study participants.

\begin{tabular}{|c|c|c|c|}
\hline & Non-overweight $(n=2683)$ & Overweight $(n=485)$ & $\boldsymbol{P}^{\mathbf{a}}$ \\
\hline Boys (\%) & 50.5 & 59.6 & $<0.001$ \\
\hline Age (years) & $9.4 \pm 0.5$ & $9.3 \pm 0.5$ & 0.534 \\
\hline Birth weight (g) & $3053.8 \pm 436.6$ & $3146.8 \pm 455.2$ & $<0.001$ \\
\hline$<2000(\mathrm{~g})(\%)$ & 1.3 & 1.5 & $<0.001$ \\
\hline $2000-2499$ & 6.9 & 4.1 & \\
\hline $2500-2999$ & 35.6 & 28.4 & \\
\hline $3000-3499$ & 41.8 & 47.0 & \\
\hline $3500+$ & 14.4 & 19.0 & \\
\hline Only child (\%) & 10.2 & 14.0 & 0.016 \\
\hline \multicolumn{4}{|l|}{ Exercise habit (\%) } \\
\hline Daily & 54.3 & 45.9 & 0.004 \\
\hline Sometimes & 26.7 & 31.3 & \\
\hline None & 19.0 & 22.7 & \\
\hline Eating snacks after dinner (\%) & 47.4 & 51.6 & 0.101 \\
\hline \multicolumn{4}{|l|}{ Eating speed (\%) } \\
\hline Fast & 15.0 & 32.7 & $<0.001$ \\
\hline Medium & 56.3 & 56.3 & \\
\hline Slow & 28.7 & 11.0 & \\
\hline Eating while doing something else (\%) & 41.0 & 43.2 & 0.391 \\
\hline Chewing thoroughly (\%) & 66.6 & 53.2 & $<0.001$ \\
\hline Skipping breakfast (\%) & 3.7 & 2.5 & 0.328 \\
\hline \multicolumn{4}{|l|}{ Dinner time (\%) } \\
\hline Regular & 96.2 & 96.0 & 0.796 \\
\hline Irregular & 3.8 & 4.0 & \\
\hline \multicolumn{4}{|l|}{ Bedtime (\%) } \\
\hline Regular & 97.1 & 95.6 & 0.117 \\
\hline Irregular & 2.9 & 4.4 & \\
\hline \multicolumn{4}{|l|}{ Wake-up time (\%) } \\
\hline Regular & 99.7 & 99.0 & 0.050 \\
\hline Irregular & 0.3 & 1.0 & \\
\hline
\end{tabular}

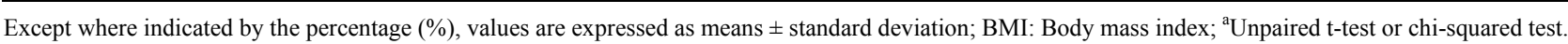

our study suggested that, when both parents are obese, not only did raising parental awareness regarding childhood obesity, but also encouraging parents to take ac- tions to reduce their own weight, led to improvements in the lifestyle habits of children. Consequently, such measures could contribute to the prevention of childhood 
Table 3. Associations between parental obesity or parental perceptions on childhood obesity and their child's overweight.

\begin{tabular}{|c|c|c|c|c|}
\hline & \multicolumn{2}{|c|}{ Crude } & \multicolumn{2}{|c|}{ Adjusted $^{\mathbf{b}}$} \\
\hline & OR & $95 \% \mathrm{CI}$ & OR & $95 \% \mathrm{CI}$ \\
\hline \multicolumn{5}{|l|}{ Parental obesity ${ }^{a}$} \\
\hline Non-obese & 1.00 & & 1.00 & \\
\hline Either parent obese & 2.02 & $1.61-2.54$ & 1.83 & $1.43-2.34$ \\
\hline Both parents obese & 5.78 & $3.83-8.73$ & 5.54 & $3.51-8.73$ \\
\hline \multicolumn{5}{|c|}{ Parental perceptions on childhood obesity } \\
\hline High & 1.00 & & 1.00 & \\
\hline Moderate & 1.59 & $1.28-1.96$ & 1.52 & $1.21-1.90$ \\
\hline Low & 2.00 & $1.46-2.74$ & 1.86 & $1.32-2.62$ \\
\hline
\end{tabular}

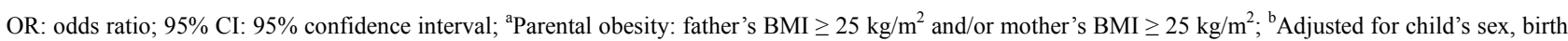
weight, existence of siblings, exercise habit, eating speed, and chewing thoroughly.

Table 4. Associations between parental perceptions on childhood obesity and their child's overweight, according to parental obesity status.

\begin{tabular}{|c|c|c|c|c|c|c|}
\hline & \multicolumn{6}{|c|}{ Parental obesity $^{\mathrm{a}}$} \\
\hline & \multicolumn{2}{|c|}{ Non-obese } & \multicolumn{2}{|c|}{ Either parent obese } & \multicolumn{2}{|c|}{ Both parents obese } \\
\hline & OR & $95 \% \mathrm{CI}$ & OR & $95 \% \mathrm{CI}$ & OR & $95 \% \mathrm{CI}$ \\
\hline \multicolumn{7}{|c|}{ Parental perceptions on childhood obesity } \\
\hline High & 1.00 & & 1.00 & & 1.00 & \\
\hline Moderate & 1.49 & $1.05-2.11$ & 1.19 & $0.79-1.80$ & 2.17 & $0.71-6.59$ \\
\hline Low & 1.93 & $1.13-3.28$ & 1.88 & $1.02-3.48$ & 1.40 & $0.20-9.57$ \\
\hline
\end{tabular}

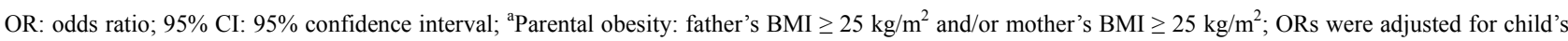
sex, birth weight, existence of siblings, exercise habit, eating speed, and chewing thoroughly.

overweight.

A limitation of the present study is that the respondent of the questionnaire was not considered. The influence of a perception about childhood obesity on overweight/ obesity, health, and lifestyle of their children may differ according to the respondent (i.e. father, mother, or other guardians). In fact, the mother's influence on the overweight of her children has been previously shown to be particularly strong $[15,34,35]$, while the father's, but not mother's, parenting behaviors and styles were associated with an increased risk of childhood overweight/obesity [36]. In future studies, the respondents to the questionnaire should be taken into consideration. Another limitation of this study was that parental height and weight was self-reported in our study. A previous study showed that self-reported weight was lower than measured weight [37]. Consequently, parental BMI in this study may have been underestimated. As a result, the sample size of the obese fathers and mothers in our study could have been decreased, and result in loss of statistical power as shown by the wide confidence interval in the stratified analysis when both parents were obese (Table 4). Lastly, in the present study, the causal relationship could not be determined, as this was a cross-sectional study.

Since the prevalence of childhood overweight and obesity in Japan as well as in the world has been increasing, it is very important to investigate the risk factor of childhood obesity. The present study indicated that childhood overweight status was significantly associated with parental low or moderate perceptions regarding childhood obesity. However, when both parents are obese, the levels of parental perceptions regarding childhood obesity may not have a substantial effect on the overweight status of their children. Therefore, it is suggested that, when both parents are obese, not only is raising parental awareness important, but also encouraging parents to take actions to improve and prevent their own overweight status. This may lead to improvements in the lifestyle habits of children, and in turn, contribute to the prevention of childhood overweight. 


\section{ACKNOWLEDGEMENTS}

The authors would like to thank all study participants, their parents or guardians, all of the members of the Board of Education in the town of Ina, Saitama Prefecture, and the Ina-machi Conference for the promotion and implementation of the Childhood Lifestyle-Related Disease Prevention Examination (Chairman: Dr. Yoshihito Toriyama). This work was supported, in part, by the Grants-in-Aid from the Ministry of Education, Culture, Sports, Science and Technology of Japan (No. 14207020 and No. 17209024).

\section{REFERENCES}

[1] Han, J.C., Lawlor, D.A. and Kimm, S.Y. (2010) Childhood obesity. Lancet, 375, 1737-1748. doi:10.1016/S0140-6736(10)60171-7

[2] Matsushita, Y., Yoshiike, N., Kaneda, F., Yoshita, K. and Takimoto, H. (2004) Trends in childhood obesity in Japan over the last 25 years from the national nutrition survey. Obesity Research, 12, 205-214. doi:10.1038/oby.2004.27

[3] Burke, V., Beilin, L.J., Simmer, K., Oddy, W.H., Blake, K. V., Doherty, D., Kendall, G.E., Newnham, J.P., Landau, L. I. and Stanley, F.J. (2005) Predictors of body mass index and associations with cardiovascular risk factors in Australian children: A prospective cohort study. International Journal of Obesity, 29, 15-23. doi:10.1038/sj.ijo.0802750

[4] Katzmarzyk, P.T., Pérusse, L., Malina, R.M., Bergeron, J., Després, J.P. and Bouchard, C. (2001) Stability of indicators of the metabolic syndrome from childhood and adolescence to young adulthood: The Québec family study. Journal of Clinical Epidemiology, 54, 190-195. doi:10.1016/S0895-4356(00)00315-2

[5] Eisenmann, J.C., Wickel, E.E., Welk, G.J. and Blair, S.N. (2005) Relationship between adolescent fitness and fatness and cardiovascular disease risk factors in adulthood: The Aerobics Center Longitudinal Study (ACLS). American Heart Journal, 149, 46-53. doi:10.1016/j.ahj.2004.07.016

[6] Stunkard, A.J., Foch, T.T. and Hrubec, Z. (1986) A twin study of human obesity. The Journal of the American Medical Association, 256, 51-54. doi:10.1001/jama.1986.03380010055024

[7] Lissner, L. and Heitmann, B.L. (1995) Dietary fat and obesity: evidence from epidemiology. European Journal of Clinical Nutrition, 49, 79-90.

[8] Sun, Y., Sekine, M. and Kagamimori, S. (2009) Lifestyle and overweight among Japanese adolescents: The toyama birth cohort study. Journal of Epidemiology, 19, 303-310. doi:10.2188/jea.JE20080095

[9] Maffeis, C., Grezzani, A., Perrone, L., Del Giudice, E.M., Saggese, G. and Tatò, L. (2008) Could the savory taste of snacks be a further risk factor for overweight in children? Journal of Pediatric Gastroenterology and Nutrition, 46, 429-437. doi:10.1097/MPG.0b013e318163b850

[10] Ievers-Landis, C.E., Storfer-Isser, A., Rosen, C., Johnson, N.L. and Redline, S. (2008) Relationship of sleep parameters, child psychological functioning, and parenting stress to obesity status preadolescent children. Journal of Development and Behavioral Pediatrics, 29, 243-252. doi:10.1097/DBP.0b013e31816d923d

[11] Jacobi, C., Schmitz, G. and Agras, W.S. (2008) Interactions between disturbed eating and weight in children and their mothers. Journal of Development and Behavioral Pediatrics, 29, 360-366. doi:10.1097/DBP.0b013e31817dbd7d

[12] Vanhala, M.L., Keinänen-kiukaanniemi, S.M., Kaikkonen, K.M., Laitinen, J.H. and Korpelainen, R.I. (2011) Factors associated with parental recognition of a child's overweight status-A cross sectional study. BMC Public Health, 11, 665. doi:10.1186/1471-2458-11-665

[13] Johannsen, D.L., Johannsen, N.M. and Specker, B.L. (2006) Influence of parents' eating behaviors and child feeding practices on children's weight status. Obesity (Silver Spring), 14, 431-439. doi:10.1038/oby.2006.57

[14] Clark, H.R., Goyder, E., Bissell, P., Blank, L. and Peters, J. (2007) How do parents' child-feeding behaviors influence child weight? Implications for childhood obesity policy. Journal of Public Health, 29, 132-141. doi:10.1093/pubmed/fdm012

[15] Birch, L.L. and Fisher, J.O. (2000) Mothers' child-feeding practices influence daughters' eating and weight. The American Journal of Clinical Nutrition, 71, 1054-1061.

[16] Arredondo, E.M., Elder, J.P., Ayala, G.X., Campbell, N., Baquero, B. and Duerksen, S. (2006) Is parenting style related to children's healthy eating and physical activity in Latino families? Health Education Research, 21, 862871. doi:10.1093/her/cyl110

[17] Dietz, W.H. (1991) Factors associated with childhood obesity. Nutrition, 7, 290-291.

[18] Eckstein, K.C., Mikhail, L.M., Ariza, A.J., Thomson, J.S., Millard, S.C., Bonns, H.J. and Pediatric Practice Research Group (2006) Parents' perceptions of their child's weight and health. Pediatrics, 117, 681-690. doi:10.1542/peds.2005-0910

[19] Maynard, L.M., Galuska, D.A., Blanck, H.M. and Serdula, M.K. (2003) Maternal perceptions of weight status of children. Pediatrics, 111, 1226-1231.

[20] Wake, M., Salmon, L., Waters, E., Wright, M. and Hesketh, K. (2002) Parent-reported health status of overweight and obese Australian primary school children: A cross-sectional population survey. International Journal of Obesity and Related Metabolic Disorders, 26, 717-724. doi:10.1038/sj/ijo/0801974

[21] Kanda, A., Kamiyama, Y. and Kawaguchi, T. (2004) Association of reduction in parental overweight with reduction in children's overweight with a 3-year follow-up. Preventive Medicine, 39, 369-372. doi:10.1016/i.ypmed.2004.01.033

[22] Shirasawa, T., Shimada, N., Ochiai, H., Ohtsu, T., Hoshino, H., Nishimura, R., Morimoto, A., Tajima, N. and Kokaze, A. (2010) High blood pressure in obese and nonobese Japanese children: Blood pressure measurement is necessary even in nonobese Japanese children. Journal of Epidemiology, 20, 408-412. doi:10.2188/jea.JE20090207 
[23] Ochiai, H., Shirasawa, T., Nishimura, R., Morimoto, A., Shimada, N., Ohtsu, T., Kujirai, E., Hoshino, H., Tajima, N. and Kokaze, A. (2010) Relationship of body mass index to percent body fat and waist circumference among schoolchildren in Japan - The influence of gender and obesity: A population-based cross-sectional study. BMC Public Health, 10, 493. doi:10.1186/1471-2458-10-493

[24] Nishimura, R., Sano, H., Matsudaira, T., Morimoto, A., Miyashita, Y., Shirasawa, T., Kokaze, A. and Tajima, N. (2009) Changes in body mass index, leptin and adiponectin in Japanese children during a three-year followup period: A population-based cohort study. Cardiovascular Diabetology, 8, 30. doi:10.1186/1475-2840-8-30

[25] Cole, T.J., Bellizzi, M.C., Flegal, K.M. and Dietz, W.H. (2000) Establishing a standard definition for child overweight and obesity worldwide: International survey. $B M J$, 320, 1240-1243. doi:10.1136/bmj.320.7244.1240

[26] World Health Organization (2000) Obesity: Preventing and managing the global epidemic. Report of a WHO consultation. World Health Organization Technical Report Series, 849, 1-253.

http://www.who.int/nutrition/publications/obesity/WHO TRS 894/en/

[27] Panagiotakos, D.B., Papadimitriou, A., Anthracopoulos, M.B. and Konstantinidou, M., Antonogeorgos, G., Fretzayas, A. and Priftis, K.N. (2008) Birthweight, breastfeeding, parental weight and prevalence of obesity in schoolchildren aged 10 - 12 years, in Greece; the Physical Activity, Nutrition and Allergies in Children Examined in Athens (PANACEA) study. Pediatrics International, 50, 563-568. doi:10.1111/j.1442-200X.2008.02612.x

[28] Wang, H., Sekine, M., Chen, X., Kanayama, H., Yamagami, T. and Kagamimori, S. (2007) Sib-size, birth order and risk of overweight in junior high school students in Japan: Results of the Toyama Birth Cohort Study. Preventive Medicine, 44, 45-51. doi:10.1016/j.ypmed.2006.07.015

[29] Maruyama, K., Sato, S., Ohira, T., Maeda, K., Noda, H., Kubota, Y., Nishimura, S., Kitamura, A., Kiyama, M., Okada, T., Imano, H., Nakamura, M., Ishikawa, Y., Kurokawa, M., Sasaki, S. and Iso, H. (2008) The joint impact on being overweight of self reported behaviours of eating quickly and eating until full: Cross sectional survey. $B M J, 337$, a2002. doi:10.1136/bmj.a2002
[30] Suka, M., Sugimori, H., Yoshida, K., Sekine, M., Yamagami, T. and Kagamimori, S. (2002) Parental influence on the development of obesity in 9-year-old Japanese children: The Toyama birth cohort study. Environmental Health and Preventive Medicine, 7, 173-175. doi:10.1007/BF02897947

[31] Burke, V., Beilin, L.J. and Dunbar, D. (2001) Family lifestyle and parental body mass index as predictors of body mass index in Australian children: A longitudinal study. International Journal of Obesity and Related Metabolic Disorders, 25, 147-157. doi:10.1038/sj.ijo.0801538

[32] Lissau, I. and Sørensen, T.I. (1994) Parental neglect during childhood and increased risk of obesity in young adulthood. Lancet, 343, 324-327. doi:10.1016/S0140-6736(94)91163-0

[33] Akhtar-Danesh, N., Dehghan, M., Morrison, K.M. and Fonseka, S. (2011) Parents' perceptions and attitudes on childhood obesity: A Q-methodology study. Journal of the American Academy of Nurse Practitioners, 23, 67-75. doi:10.1111/j.1745-7599.2010.00584.x

[34] Mizutani, T., Suzuki, K., Kondo, N. and Yamagata, Z. (2007) Association of maternal lifestyles including smoking during pregnancy with childhood obesity. Obesity, 15, 3133-3139. doi:10.1038/oby.2007.373

[35] Adamo, K.B., Papadakis, S., Dojeiji, L., Turnau, M., Simmons, L., Parameswaran, M., Cunningham, J., Pipe, A.L. and Reid, R.D. (2010) Using path analysis to understand parents' perceptions of their children's weight, physical activity and eating habits in the Champlain region of Ontario. Paediatrics and Child Health, 15, e33-41. http://www.ncbi.nlm.nih.gov/pmc/articles/PMC3009571/

[36] Wake, M., Nicholson, J.M., Hardy, P. and Smith, K. (2007) Preschooler obesity and parenting styles of mothers and fathers: Australian national population study. Pediatrics, 12, e1520-1527. doi:10.1542/peds.2006-3707

[37] Del Duca, G.F., González-Chica, D.A., Santos, J.V., Knuth, A.G., Camargo, M.B. and Araújo, C.L. (2012) Self-reported weight and height for determining nutritional status of adults and elderly: Validity and implications for data analysis. Cadernos de Saúde Pública, 28, 75-85. doi:10.1590/S0102-311X2012000100008 